\title{
Sturge Weber Syndrome Suggested by Observation of Plantar Hemangioma
}

\author{
Hallaung Masoj \\ Department of Neurology, Fann Teaching hospital, Dakar-Senegal
}

\begin{abstract}
Sturge -weber krabbe syndrome is a very rare neurocutaneous and ocular phacomatosis with a malformative vascular substratum, the skin involvement of which is often unilateral affecting the territory of the trigeminal nerve. Here we report a case with plantar angiomas associated with angioma of the face.
\end{abstract}

Keywords: Plantar angioma, STURGE WEBER

\section{Introduction}

Also called, Sturge weber Dimitri syndrome or Encephalotrigeminal angiomatosis is a neuro-oculocutaneous syndrome associating a facial angioma; a leptomenal angioma and an ocular abnormality (Enjolras et al)

\section{Case Report}

2-year-old patient; of second-degree consanguineous parents from a twin pregnancy. He was born at term. Admitted to DIAMNADION hospital on December 24, 2020 for left hemi-body tonic convulsive seizures followed by homolateral hemi body deficit on January 28, 2020. Remission of seizures for 8 months.

\section{General clinical examination}

- Good condition; mucous membrane normal discoloration; good state of hydration.

- Presence of an angioma on the face and sole of the right foot.

- Neurological examination today found left pyramidal syndrome of the cortical type and psychomotor hyperactivity

- Biological assessment without particularity.

Brain imaging

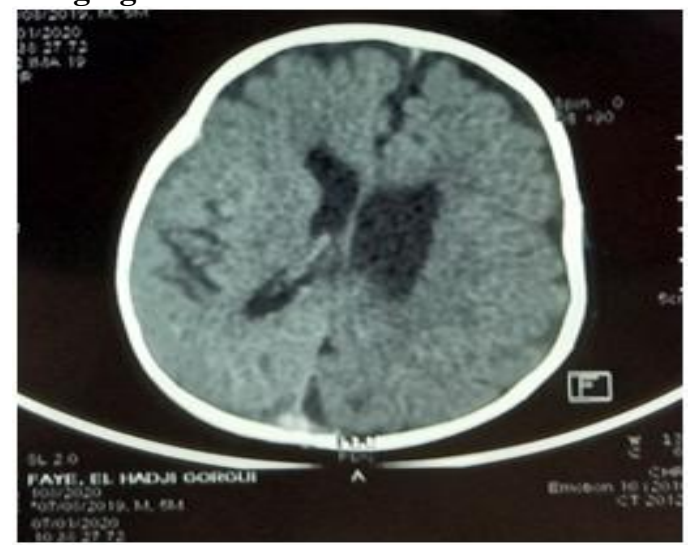

Photo: CT brain parenchymal window

Axial cut; floor above tentorial objective middle structure in place; Hemi- atrophy of the right hemisphere

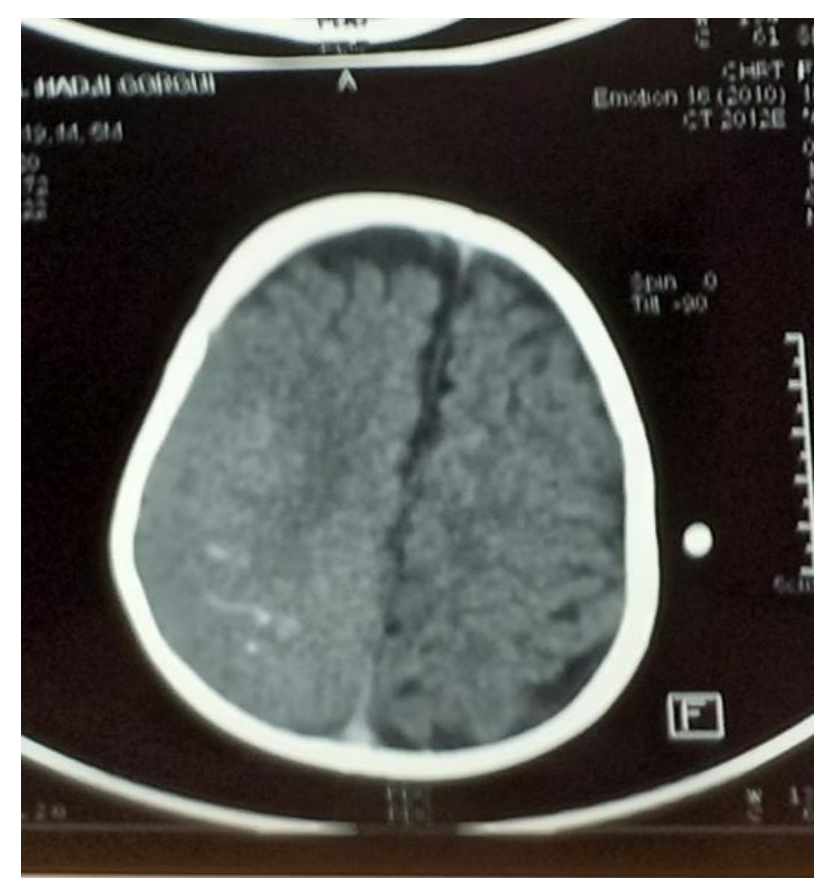

Photo: Brain CT parenchymal window axial cut above tentorial level objective cortical calcification on the left side at the right parieto-occipital level.

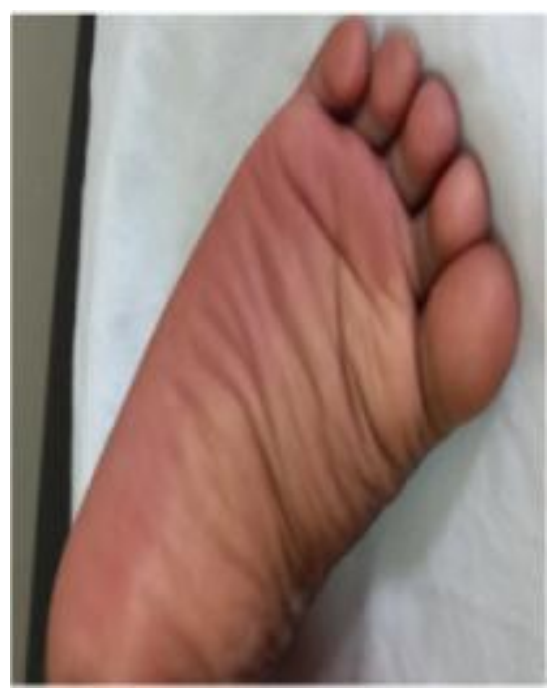

Figure: Right plantar angioma 
The electroencephalography (EEG) finds an asymmetry of the tracing secondary to the right cortical atrophy and the presence of physiological figures of light slow sleep on the left side due to the functionality of the cortex. Sleep electroencephalography: asymmetry of the monomorphic trace; devoid of the physiological figures of slow sleep on the right. On the left progressive sleep stage I; II and III with a background Delta-theta rhythm at $2-4 \mathrm{~Hz}$; irregular and polymorphic. Presence of physiological figure of light then deep slow sleep. Presence of a center point in the left frontal with diffusion to the right. Intermittent silt stimulation: No effect. Conclusion asymmetric sleep EEG tracing; well organized on the left with irritative signs on the right frontal compatible with focal epilepsy.

Epileptic treatment depends on the clinical semiology of the seizures; in our observation, we used sodium valproate at a dose of $20 \mathrm{mg} / \mathrm{kg}$ divided into two doses with amendment of the attacks.

\section{Discussion}

Sturge-Weber Krabbe syndrome is a neurocutaneous and ocular phacomatosis with a very rare vascular malformation substratum, the skin involvement of which is often unilateral, reaching the territory of the trigeminal nerve. Its presence is very suggestive of the diagnosis but its absence does not exclude it, ocular damage is dominated by glaucoma (30-70\%), a choroidal angioma is found in 40$50 \%$ of cases, the fundus will look for then a retinal liftreddish [Boukhobza $\mathbf{M}$ et al].The neurological signs are dominated by epilepsy ( 75 to $90 \%$ of cases), often early and severe, partial motor seizures of the contralateral hemi body in $70 \%$ of cases. Motor deficit as well as mental retardation are found in $50 \%$ of cases. Psychiatric disorders, which have been described, remain rare [Sujanski E et al]. Roch and Coll., which describes three forms of Sturge Weber, has proposed a classification type 1 (classic): manifestations

Intracranial and facial; Type 2: facial impairment alone without central changes; Type 3: intracranial manifestations alone. Our observation belongs to type one associated with a respective plantar involvement on the right side; both sexes are affected with a male predominance which corroborates with our observation, no mental retardation objectified on our observation. The symptomatology was constituted by right focal seizures associated with a right hemi paresis. A segmental muscle strength estimated at $3 / 5$ on the right side and $5 / 5$ on the left side. The occurrence of convulsive seizures on the left side is explained by the atrophy of the right cortex and the persistence of the fibers of the interhemispherical communications which transport epileptic discharge from the contro-lateral side by cortices on the right. Facial angioma in the trigeminal territory on its ophthalmic branch in our patient, but the association with plantar angioma differentiated from other classification proposed by $\mathrm{ROCH}$ et al.

\section{Conclusion}

Sturge weber syndrome is a rare pathology often overlooked. The »major objective is the clinical diagnosis which is based on facial angioma, cerebral damage but also search for other localization of the angioma as in the reported case here on a plantar location.

\section{References}

[1] Boukhobza M, Enjolras O, Cambra MR. JJ SturgeWeber syndrome: The current neuroradiologic data. J Radiol. 2000;81(7):765-771. [PubMed] [Google Scholar]

[2] Enjolras O, Riche MC, Merland JJ Facial port wine and Sturge Weber syndrome. Pediatrics. 1985; 76(1):48-51. [PubMed] [Google Scholar]

[3] Sarcaoglu MS, Guven D, Darakurt A, Sengun A, Ziraman I. An unsualcase of sturgeweber syndrome in association with phakomatosis pigmen to vascularis and klippeltrenaunay-weber syndrome. The Ophthalmic Communications Society. 2002; 22(3):368-371. [PubMed] [Google Scholar]

[4] Sujanski E, Conradi Sturge Weber syndrome: age of ondet of seizures and glaucoma and the prognosis for affected children. J Child Neuro. 1995 Jan; 10(1):4958. [PubMed] [Google Scholar] 